\title{
Cost Model For Establishing A Data Center
}

\author{
Doaa Bliedy, Sherif Mazen and Ehab Ezzat \\ Department of Information System, Cairo University, Cairo
}

\begin{abstract}
Servers and other IT devices inside datacenters have hardware component and other software or virtual component. Some programs are required to create and secure everything related to the virtual environment on IT devices. These programs can be free or require a pre-paid license to be accessed .Most of the proposed datacenter total cost of ownership (TCO) models focus only on calculating the costs of the hardware component of IT devices and they ignore the costs of the other virtual component.In this paper, we present a cost model for building a datacenter and provide through it an analysis way to calculate the IT software license cost. Our model helps in solving a real problem in faculty of computers and information which plans to establish a datacenter. Our model can help the faculty administrators to know how much money they need to buy the IT devices and how much IT software license cost. We also calculate the cost of the power distribution equipment(PDU) and the uninterruptable power supply (UPS) systems which are required for operating the IT devices. The cost of the cooling systems that take the heat away once the power is consumed from IT devices, PDU devices and UPS systems is also calculated in our model.
\end{abstract}

\section{KEYWORDS}

Datacenters, TCO model, TCO parameters, IT software license cost, network, server

\section{INTRODUCTION}

Data centers can be defined as any space whose main function is to house servers [6] or computing devices that are in-use, i.e., are powered on and performing functions. Although a small computing room inside a multipurpose building can be believed as a data center, the term is traditionally used to define buildings whose major function is to locate these servers. In this conventional sense, human occupancy is limited to small Information Technology (IT) support groups who may have office space within the building - these office spaces are small relative to the total size of the building. These facilities differ greatly from most buildings from a construction perspective. For example, mechanical and electrical systems account for $70 \%$ of construction costs in data centers, in contrast to only $15 \%$ of costs in commercial buildings [7]. Nowadays, network-based activities or internet are the major reasons for using datacenters. Datacenters locate servers so that they can Manipulate large amount of data, talk to each other or to other computer networks and process user interactions with server-based software tools or web portals.

The other main purpose of the existence of data centers today is the need for managing the critical data and operations such as organization's databases and email sending As a result, reliabilityi.e., its ability for the servers to be working properly and not waste data-is a critical interest for many data centers. This reliability depends on many factors such as the characteristics of the servers used and the data center "infrastructure" which contains the power delivery system and cooling resources. Servers and other computing devices need continuous power supply system and are less susceptible to hardware breakdown when operating below a certain temperature. It is significant to mention that the servers can produce large amount of heatand, as a result, data centers often have large cooling loads [8]). Facility owners often use redundant computing setups, redundant power delivery systems, Uninterruptable Power Supply (UPS) devices, and cooling resources to protect server machines from failure. 
The level of redundancy in the power generation system and cooling resources are usually used to evaluate the rank of reliability in data centers. This is known as the data center 'Tier level' [9] where higher Tier levels represent greater redundancy. Using redundant cooling resources, power delivery devices and UPS units increase the electrical energy consumed by the facilities. On the other hand, some buildings don't need such redundancies and equipment. As internet-based services have become increasingly used, datacenter establishment has increased. Through the period 2000 to 2010, the yearly establishment of data centers (in terms of money spent) increased over $300 \%$, from approximately $\$ 15$ billion USD to $\$ 50$ billion USD [10]. Some of this increased spending is linked to the more facilities being constructed annually, while others are linked to the increased redundancy in the newer facilities. That is, a 'Tier 4' data center can cost $\$ 22$ million USD/MW in comparison to $\$ 10$ million USD/MW for a 'Tier 1' facility [10].

The datacenter market can be split roughly into two sub-sectors: colocation and enterprise. Colocation, or "colo" operators provide what is known colloquially as "position, power and ping" which basically means that they provide the infrastructure - security, constant electricity supply, broadband connectivity and an environment in which temperature and humidity is controlled to suit servers. They then sell or lease space within those specialized facilities to companies who install and manage their own IT equipment. The term colocation comes from the fact that these customers share or "co-locate" their IT operations in one purpose built facility. Enterprise operators are those who use their datacenter provision for their own purposes - i.e. for their own corporate IT functions (in the case of banks and supermarkets and government departments). Enterprise operators also include those who provide IT services to third parties (HP, Fujitsu, IBM, BT, Atos, CapGemini, etc). Enterprise datacenter operators may build their own datacenter facilities or locate their datacenters within colo facilities - Many companies do both. Some companies operate enterprise datacenters but also sell some colo space to other companies, and this is a logical option for enterprise operators who find themselves with spare capacity.

As mentioned above, enterprise operators may build their own datacenters but for most it makes commercial sense to use a third party provider. Once an organization's data requirements reach a certain size or become mission critical (where disruption of service has significant adverse consequences and generates liabilities), this data will need to be housed in an environment with guaranteed levels of security, continuity of power supply and connectivity. Companies have a number of options here: They could build their own facility or they could take space from a wholesale or a colo operator and still manage their IT themselves or they could outsource the whole IT function to an IT services provider (who in turn may have their own datacenter or have taken space within a colo). They could even buy a site and then contract a third party to manage their IT for them within it. In this paper we focus on the situation that the company chooses to build their in-house and own datacenter from scratch.

\subsection{Datacenter TCO Models ANd SPREAdSheet ToOlS}

In this part, we overview the proposed datacenter TCO models and spreadsheet tools .The calculations and estimations of TCO of the data centers don't have any recognized standards or rules; they depend on the size, location and design of datacenters [11]. .Many TCO models have been proposed for guiding datacenters design [1],[2], [3], [4], [5] that mainly depend on two types of datacenter costs the capital costs and operating costs for running the datacenter. Only few tools are publicly available to calculate TCO. APC [16] provides an online estimator tool while [4], [15] provide spreadsheets to estimate the TCO. These tools can be used to assess the benefits and drawbacks of datacenter design choices on the TCO and the environmental impact but they do not allow easy exploration and fine grain design choices.

Based on these TCO models and tools, we can divide Datacenter environment into number of IT equipment and number of operating devices required for operating the IT equipment. We collect the different devices and parameters in the datacenter and show them in Table [1]. 
Table 1. The Different devices in Datacenter.

\begin{tabular}{|c|c|}
\hline IT Equipment & Operating devices and systems for IT equipment \\
\hline $\begin{array}{ll}\text { - } & \text { Server machines } \\
\text { - } & \text { storage equipment } \\
\text { - } & \text { Networking machines }\end{array}$ & $\begin{array}{ll}\text { - } & \text { Power delivery and generation systems including (UPS } \\
\text { - } & \text { systems and power distribution units (PDU)) } \\
\text { - } & \text { Fire protection systems } \\
\text { - } & \text { Raised floor / dropped ceiling (may be needed or not) } \\
\text { - } & \text { enclosures and containment (Racks) } \\
\text { - } & \text { Lighting system }\end{array}$ \\
\hline
\end{tabular}

The TCO models and spread sheet tools also helped us to know the different kinds of expenses in datacenters. The TCO of a datacenter includes Capital expenses and other Operational expenses. We show the different costs in datacenters in Table [2].

Table 2. The Different Kinds of Expenses in Datacenters.

\begin{tabular}{|c|c|}
\hline Capital cost & Operation cost \\
\hline $\begin{array}{l}\text { - hardware price (acquisition cost of IT } \\
\text { devices, the power distribution and } \\
\text { cooling equipment and other } \\
\text { operating systems acquisition cost ) } \\
\text { - basic installation, Design } \\
\text { /engineering costs } \\
\text { - land cost } \\
\text { - building cost } \\
\text { - project management / facility } \\
\text { engineering cost }\end{array}$ & $\begin{array}{l}\text { - Power consumption cost (the cost of } \\
\text { electricity for servers, networking equipment } \\
\text { and cooling.) } \\
\text { - } \quad \text { Personnel cost (personnel salaries) } \\
\text { - } \quad \text { Maintenance and repairs costs } \\
\text { - } \quad \text { Depreciation (amortization) costs) } \\
\text { - IT software license cost }\end{array}$ \\
\hline
\end{tabular}

Unfortunately, none of the previous models or spread sheet tools provide a step by step an analysis way to calculate and estimate the software license cost, they focus only on calculating and estimating costs of the physical hardware side of servers and other IT devices and they ignore the costs of the other virtual side of the IT devices. In our paper, we build accost model for buildinga datacenter and provide through it an analysis way to calculate the IT software license cost. Our model helps in solving a real problem in faculty of computers and information which locates in Fayoum University. The faculty has many problems in their computer devices and plans to establish a new datacenter. That cost model can help the faculty administrators to define and know how much money they need to buy the required IT devices and how much IT software license cost. We also calculate the cost of the powerdistribution equipment required to provide power to IT devices and the cost of the cooling systems that take the heat away once the power is consumed from IT devices.

The rest of the paper is organized as follows. Section 2 overviews the problem definition and the proposed solution, while The Data and methods of the proposed model are given in Section 3. Results are discussed in section 4. Finally, section 5 concludes the paper and gives directions for future work.

\section{Problem Definition}

Faculty of computers and information is an Egyptian faculty .The faculty building has five computer labs; each computer lab contains 24 desktop computer devices with a total number of 120 computer devices. These computer labs are dedicated for students for their practical research activities. 
Each computer device has its own operating system (one OS per machine) and some existing installed software applications. The computer device has some basic physical resources such as CPU, memory, hard disk and the network resources. We show in Figure [1] the hardware and software component of the desktop computer devices in the faculty.

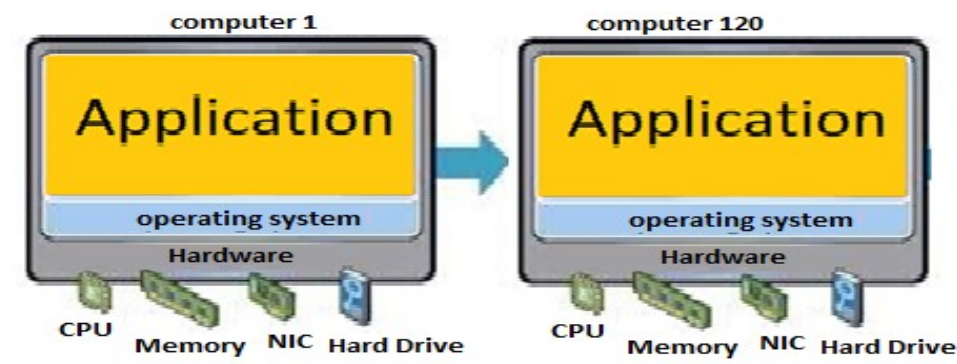

Figure 1. Computer device structure

The main problem is that the students who use the computer devices in the faculty may use some virus infected files and download insecure and untrusted applications on computer devices. These files and applications will have a bad effect on the performance of the computer operating system we think about a solution for that problem is to buy another 120 desktop computer and allow the student to work on two computer devices, one device for the practical research activities and the other computer for the untrusted applications and that solution is difficult to be applied in the faculty because the new computer devices will require more space and there is no more available space in the faculty for locating them, besides that, it is difficult for the student to work on two devices in the same time. We think about another solution is to enable Virtualization technology on each computer device. We mean by Virtualization technology is to create number of virtual machines on each computer device and the untrusted or unsecured application can be opened and run inside the virtual machine and that will not affect the host machine.

Using the virtual machines has numerous benefits for the students, being able to essentially have another computer running within an operating system allows for tasks such as software testing, running applications on outdated or incompatible operating systems and even allows for the opening and downloading of virus infected files, without the risk of infecting the host machines. In details, creating Virtual machines on computer devices have a great array of uses for the students. Here are the reasons for using virtual machines:

- Test with different operating systems

The user can install different distributions of Linux or other lesser known operating systems in a virtual machine to experiment with them and to understand how they work. Multiple operating systems can run in the same computer, eliminating the need to dedicate a single machine to one application

- Utilizing software that requests an outdated operating system

$\mathrm{n}$ a situation where the users have an important application that only runs on Windows XP, they can install XP in a virtual machine and run that rather prehistoric application in the virtual machine where compatibility isn't an issue. The best part is that virtual machines allow the user to use an application that only works with Windows XP without actually installing Windows XP on the computer which is definitely handy since not many hardware fully support Windows XP.

- Run software formatted for other operating systems

Mac and Linux users can also run Windows in a virtual machine to run Windows software on their computers without the compatibility headaches of Wine and Crossover. 
In general, all these uses are the reasons for creating virtual machines on computer devices but in our model, we need to create the virtual machine to protect the desktop computer device. Creating virtual machine on the computer device will provide the students with the opportunity to open and run the untrusted files inside the virtual machine, without the risk of infecting the host machines. VMs are "sandboxed" from the rest of the computer system. Software inside a VM can't escape the VM to tamper with the rest of the computer system. This makes VMs a safe place to test apps - or websites - the students don't trust and see what they do. The following figure shows the architecture of computer device before and after virtualization

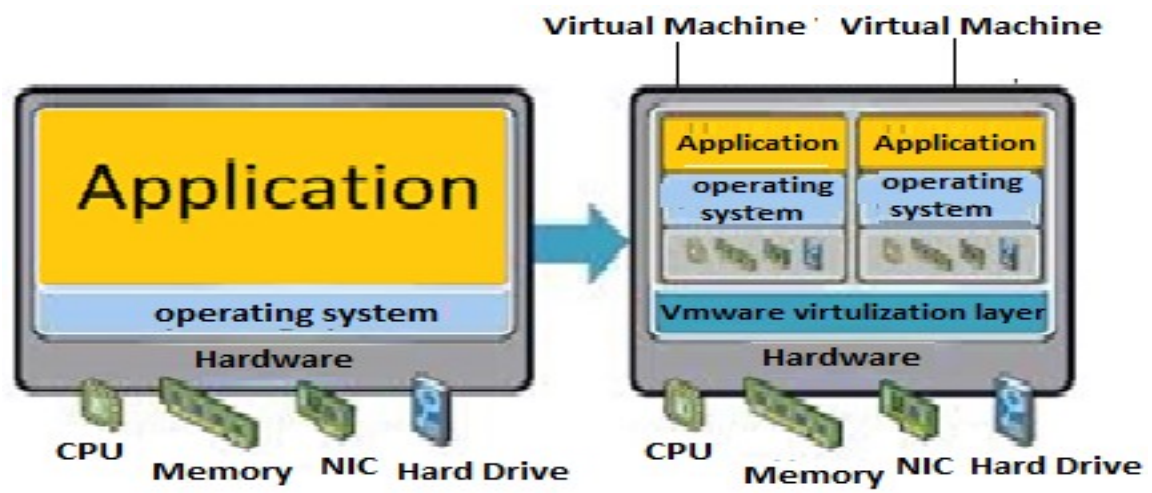

Figure 2. the architecture of computer device before and after virtualization

Before virtualization, only one operating system can be installed per computer machine but after virtualization, more than one operating system can be installed on the same computer device. The virtual machine is like a separate computer, it has its own operating system and applications. VM's operating system is isolated from the host operating system. The students can open and test any untrusted files within the virtual machines without affecting the host machine.

Some virtual machine apps and programs are required to be installed on computer devices to create the virtual machines such as hypervisor, Vmware, Virtual PC, VirtualBox or Qemu. This Specialized software, called a hypervisor, emulates the computer device's CPU, memory, hard disk, network and other hardware resources completely, enabling virtual machines to share the resources. The hypervisor can emulate multiple virtual hardware platforms that are isolated from each other, allowing virtual machines to run for example " Linux and Windows Server operating systems" on the same underlying physical machine. Once the hypervisor is installed, a virtual machine can be considered a "container" that can be arbitrarily backed up, and used by students. Other programs are required to manage the process of running and securing everything related to the virtual environment on computers.

The VM runs as a process in a window on the current operating system of computer devices. The users can boot an operating system installer disc (or live $\mathrm{CD}$ ) inside the virtual machine, and the operating system will be "tricked" into thinking it's running on a real computer. It will be installed and run just as it would on a real, physical machine. Whenever the users want to use the virtual machine, they can open the virtual machine program and use it in a window on the current desktop computer.

In the VM world, the operating system actually running on the computer is called the host and any operating systems running inside VMs are called guests. In a particular VM, the guest OS is stored on a virtual hard drive - a big, multi-gigabyte file stored on the real hard drive. The VM app presents this file the guest OS as a real hard drive. This means the users won't have to mess around with partitioning or doing anything else complicated with the real hard drive.

The limit to how many VMs the users can create on the computer devices are really just limited by the amount of processor speed (CPU cores), the memory size, the amount of hard drive space 
and the amount of available network resources .the users can also run multiple VMs at the same time, but they'll find themselves somewhat limited by the computer's system physical resources. Each VM eats up some CPU time, RAM, and other resources. The physical computer must have enough memory size, CPU cores, hard disk space, and network resources to accommodate both the host operating system and the guest operating system (VM OS).

The problem in the faculty is that the computer devices are old and are not powerful computers to begin with virtualization. The computers don't have enough processor cores, memory size, and storage space and network resources to successfully install the VM apps and in the same time they aren't suitable and ready for running more than one operating system in the same time.

this problem can be solved by ,Instead of creating virtual machines on the computer devices themselves, they can be created on remote server and accessed by the student in the faculty at any time. The faculty administrators can buy number of server machines and virtualizing their resource and allocating for each computer device a virtual machine. The virtual machine created on the server machines can be accessed by the student (user of the computer device) at any time. In Figure [3], we show the connection between the computer device in the faculty and the virtual machine which will be created on the server machines.

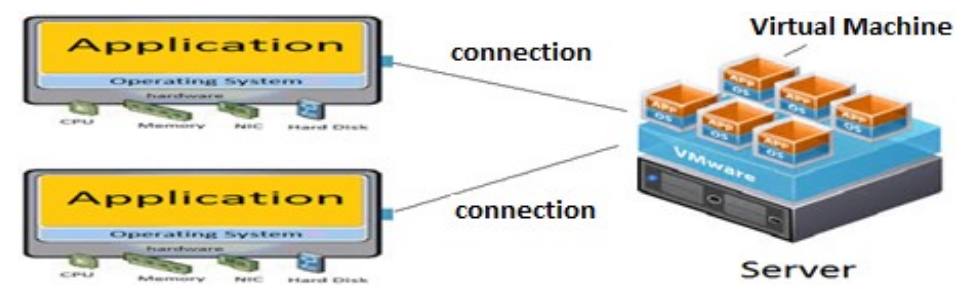

Figure 3. the connection between computer devices and servers

The faculty administrator should establish datacenter building to house the server machines and provide all the devices required for operating and securing servers and their virtual machines. There are many companies that offer solutions for setting up private data centers or renting data centers over the Internet (eg EMC2, Amazon, IBM, Oracle, etc ...). The requirements for creating or leasing data centers differ in terms of the financial cost and each model has its advantages and disadvantages. In our model, we present cost model that can be used by the faculty administrators to determine how much money they need to establish the datacenter.in our cost model, we are calculating the IT devices cost, IT software license cost, the cost of the power distribution equipment required to provide power to IT devices and the cost of the cooling systems that take the heat away once the power is consumed from IT devices, PDU devices and UPS systems.

\section{DATA AND Methods}

\subsection{IT DeVices (SERVers, SAN Storage ANd Networking Machines HaRdware REQUIREMENTS)}

\subsubsection{Virtual machines hardware requirements}

Determining the required amount of physical hardware resources of a server is depending on the number of virtual machines that will be created on the server and the applications that are planned to be installed on it. As we mentioned before, in our model, the students only need a separate computer device to open the untrusted websites or applications. These untrusted applications can be opened and run on the computer devices themselves but they will have a bad effect on the computer device, so we can create virtual machine on server with the same physical hardware specifications of the computer device and that virtual machine will be accessed by the student at any time, the students will see and deal with the virtual machine as an adjacent computer. 
International Journal of Computer Science, Engineering and Applications (IJCSEA) Vol. 8, No. 2/3/4, August 2018

The computer device in the faculty has some physical hardware resources. We show in Table [3] the physical hardware resources of one computer device. In our model, for each computer device, a virtual machine will be created with the same physical hardware resources.

Table 3. Virtual machine physical hardware resources

\begin{tabular}{|c|c|}
\hline Component & Specifications \\
\hline CPU & CPU with 2 core \\
\hline RAM & 8 GB \\
\hline Network card & $1 \mathrm{~Gb} / \mathrm{s}$ Ethernet card \\
\hline Hard Disk & 250 GB hard disk \\
\hline
\end{tabular}

By calculating these hardware specifications for 120 virtual machines working at the same time, we need servers with the following hardware specifications:

Table4. Total servers hardware requirements

\begin{tabular}{|c|c|}
\hline Component & Specifications \\
\hline CPU & $120 * 2$ core $=240$ core \\
\hline RAM & $120 * 8 \mathrm{~GB}=960 \mathrm{~GB}$ \\
\hline Network card & $120 * 1 \mathrm{~Gb} / \mathrm{s}$ Ethernet \\
\hline HARD DISK & $120 * 250 \mathrm{~GB}$ hard disk $=30,000 \mathrm{~GB} \sim 30 \mathrm{~TB}$ \\
\hline
\end{tabular}

These hardware specifications can be provided with 10 servers that are connected in a workgroup with hardware specifications for each server as follows:

Table5. One server hardware requirements

\begin{tabular}{|c|c|}
\hline Component & Specifications \\
\hline CPU & 24 core \\
\hline RAM & $96 \mathrm{~GB}$ \\
\hline Network card & $10 \mathrm{~Gb} / \mathrm{s}$ full duplex card \\
\hline HARD DISK & $3000 \mathrm{~GB}=3 \mathrm{~TB}$ \\
\hline
\end{tabular}

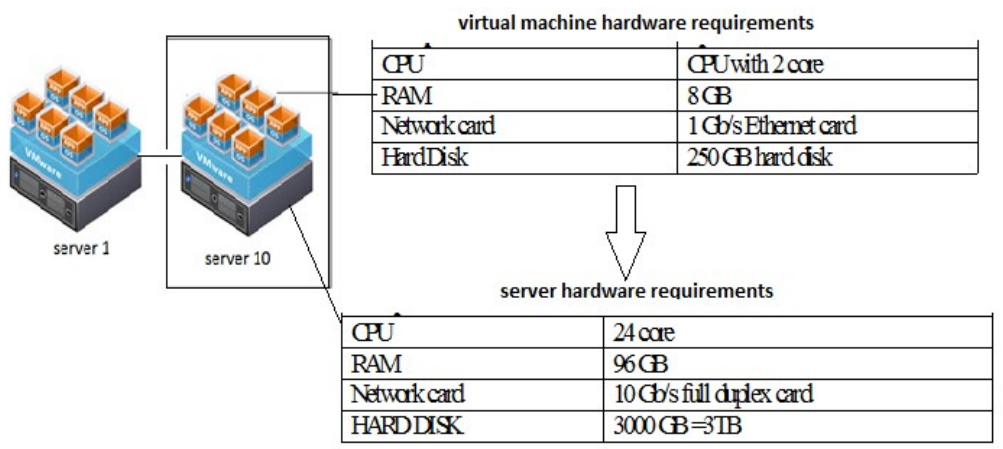

Figure 4. Server hardware requirements

We show in figure [4] the virtual machine and the server hardware requirements. For our storage system requirements, we need to allow the storage to be accessible and shared by all servers so we need to buy SAN Storage. We need to increase the performance and reliability of data storage so we will deploy RAID technology (Redundant Array of Inexpensive Disks).A RAID system consists of two or more drives working in parallel.

We need to allocate more storage disks as backup drives for servers, we will use RAID 5 technology. RAID 5 arrays can withstand a single drive failure without losing data or access to data. We assume that the single disk storage size is one tera byte (1TB), so each server will need 3 disks with a total storage size of $3 \mathrm{~TB}$, to enable RAID 5 arrays technology we need to provide additional one (1TB) hard disk storage to work as a backup hard drive . 
International Journal of Computer Science, Engineering and Applications (IJCSEA) Vol. 8, No. 2/3/4, August 2018

For each server, Number of disks is 4 disks with a Single disk size $=1$ TB so Total Capacity of RAID 5 disks is 3TB with Fault tolerance of 1 drive failure. .

We need to buy SAN Storage with a total capacity of $40 \mathrm{~TB}$. We show in figure [5] the servers and SAN storage device architecture.

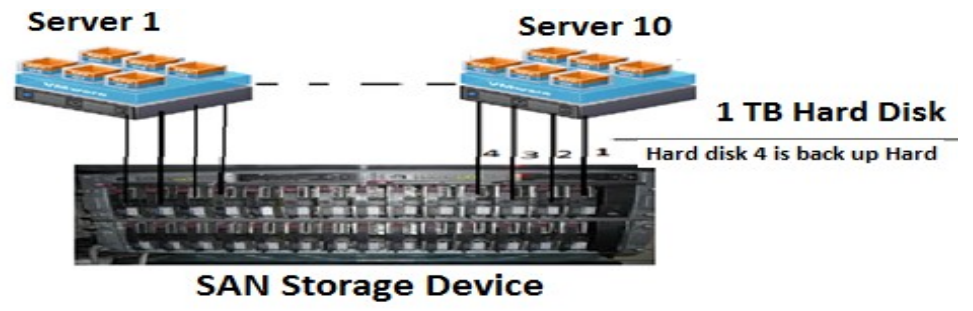

Figure 5. Servers and SAN storage device architecture

\subsubsection{APPLICATIONS HARDWARE REQUIREMENTS}

Some programs are required to manage the servers and organize the process of transferring data between them as well as managing the process of creating, running, securing and Operating everything related to virtual machines. These applications or programs have some hardware requirements so we need to provide more CPU, memory, storage and network resources to meet the minimum system requirements of installed applications for example, Windows Server 2012 R2 Hardware Requirements are 2 GB RAM or greater and 40 GB or greater of Available Disk Space, after checking the applications system requirements for Windows Server 2012 R2 [14],ESXI vcenter server 6.5 [17], Oracle DB 11g R2 [12] and Veeam Backup \& Replication 9.5 [13],that are available on the vendor websites to be installed on servers, we have to allocate more hardware resources to meet the applications system requirements and specifications. So we add the applications systems hardware requirements and the virtual machines hardware requirements to get the total server hardware resources requirements, we need 10 servers that are connected in a workgroup with specifications for each server as follows:

Table6. Server hardware requirements

\begin{tabular}{|c|c|}
\hline Component & Specifications \\
\hline CPU & 28 core \\
\hline RAM & $176 \mathrm{~GB}$ \\
\hline Network card & $10 \mathrm{~Gb} / \mathrm{s}$ full duplex cards (two cards) \\
\hline HARD DISK & (40 TB SAN Storage device $)+(300 \mathrm{~GB}$ for applications $)$ \\
\hline
\end{tabular}

Servers and SAN storage device hardware price differs from one vendor and others; we can buy our machines from HP, DELL, IBM or other companies. Server hardware price depends on the number of CPU cores, Memory size, storage space and their type.in table [7], we illustrate the server resources types that we can use in our model. After checking the online technical datasheets of several servers, we found that "ProLiant ML350 Gen10 SFF server "introduced from HP Company can meets our requirements. More technical details about the server is illustrated in [18]. Our servers can be accessed and monitored by using (HPE LCD8500 KVM Console Switch). The SAN storage hardware price depends on the storage capacity. We searched many times about the available SAN storage devices over different vendors websites and we found that "HP StoreServ 7400c 4N" SAN Storage is suitable to be used in our datacenter.in table [8], we show the different hardware components of the SAN storage device including the number of hard disks and their storage capacity. More information about the SAN Storage devices is illustrated at [19]. 
International Journal of Computer Science, Engineering and Applications (IJCSEA) Vol. 8, No. 2/3/4, August 2018

Table7. Server machine (hpproliant m1350 gen10 sff)

\begin{tabular}{|c|c|c|c|c|}
\hline Component & Specification & Type & $\begin{array}{c}\text { Part } \\
\text { Number }\end{array}$ & $\begin{array}{c}\text { Quanti } \\
\text { ty }\end{array}$ \\
\hline Processor & HPE ML350 Gen10 8180 Xeon-P Kit & $28 \mathrm{CORE}$ & $\begin{array}{c}878662- \\
\text { B21 }\end{array}$ & 1 \\
\hline Memory & HPE 16GB 1Rx4 PC4-2666V-R Smart Kit & DDR-4 & $\begin{array}{c}815098- \\
\text { B21 }\end{array}$ & 11 \\
\hline Storage & HPE 300GB SAS 10K SFF SC HDD & Hard Drive & $\begin{array}{l}\text { 785067- } \\
\text { B21 }\end{array}$ & 1 \\
\hline Expansion & HP Ethernet 10Gb 2P 530T Adptr & $\begin{array}{c}\text { PCIe } \\
\text { (Network } \\
\text { Adapter ) } \\
\end{array}$ & $\begin{array}{l}\text { 656596- } \\
\text { B21 }\end{array}$ & 2 \\
\hline $\begin{array}{c}\text { Expansion } \\
\text { For (iSCSI } \\
\text { SAN storage) }\end{array}$ & HP Ethernet 10Gb 2P 530SFP+ Adptr & $\begin{array}{c}\text { PCIe } \\
\text { (Network } \\
\text { Adapter ) } \\
\end{array}$ & $\begin{array}{c}\text { 652503- } \\
\text { B21 }\end{array}$ & 1 \\
\hline Power supply & HPE 1600W FS Plat HtPlg LH PwrSply Ki & & $\begin{array}{c}830272- \\
\text { B21 }\end{array}$ & 1 \\
\hline Fans & $\begin{array}{l}2 \text { standard fans; Optional redundant fan } \\
\text { cage kit (874572-B21, add' } 14 \text { fans) }\end{array}$ & & & 6 \\
\hline
\end{tabular}

Table 8. SAN storage components

\begin{tabular}{|c|c|c|}
\hline \multicolumn{3}{|c|}{ HP StoreServ 7400c 4N -QR513A } \\
\hline Model Name & PartNumber & Quantity \\
\hline \multicolumn{2}{|c|}{ Storage } \\
\hline HP M6710 1TB 6G SAS 7.2K 2.5in HDD & QR498A & 40 \\
\hline \multicolumn{2}{|c|}{ Expansion } \\
\hline HP 3PAR 7000 2-pt 10Gb Eth Adapter & E7X96A \\
\hline \multicolumn{2}{|c|}{ Power Supply } \\
\hline POWER COOLING MODULE 764W & 682372-001 & 4 \\
\hline
\end{tabular}

\subsubsection{NETWORKING MACHINE HARDWARE REQUIREMENTS}

After knowing the number of servers and the SAN storage device, we need to design LAN network for the datacenter to enable servers to communicate with each other and communicate with the SAN storage device. To set up an Ethernet LAN inside our datacenter, we need some devices such as switches, patch panels, network interface cards, patch cables and number of fiber optical cables. The Switch acts as a connecting point between servers on a network. We need switch with the following specifications

Table9. Switch specification

\begin{tabular}{|c|c|}
\hline Component & Value \\
\hline Speed & $10 \mathrm{~Gb} / \mathrm{s}$ full duplex \\
\hline Ports & 48 port \\
\hline link aggregation & TRUE \\
\hline Vlans (Virtual LAN ) & TRUE \\
\hline console interface/programble & TRUE \\
\hline
\end{tabular}

After checking the datasheet of different network switches available on different company's websites, we found that "Dell Networking N4064" switch matches our requirements .the technical datasheet of the switch is available at [17]. The main function of the switch is to handle network traffic, receive and direct packets of data across the LAN.A network interface cards (NIC) will be installed in each server, and will be assigned to a unique address. We have 10 servers in our datacenter; our plan is to install (HP Ethernet 10Gb 2P 530T Adptr) in each server. The network card has ports that can accommodate cables for connection. 
International Journal of Computer Science, Engineering and Applications (IJCSEA) Vol. 8, No. 2/3/4, August 2018

About cables, 10GBASE-T Network can be run on Category 6A cables. An (Pro snake CAT6a Patch Cable SFTP) will be run from each NIC port in the server to patch panel and other patch cable from the patch panel to the switch. Besides enabling the communications between servers, we need SAN switch to connect between the servers and the SAN storage device.

There are three primary iSCSI SAN hardware components. These include iSCSI storage NICs (dedicated network interface cards for iSCSI communications) or initiators; standard Ethernet network switches; and target storage NICs or targets on the external storage arrays.

In our model, we plan to install (HPE Ethernet 10Gb 2-port 530SFP+ Adapter )in each server, they are iSCSI initiators .Our servers HBA cards will be connected to iSCSI SAN switch and the switch will be connected to iscsi HBA in SAN storage device. Cabling (Fiber optical cables (multi-mode cables)) will be used to connect the HBA's port to a corresponding port on a switch. Each storage server and storage device connects to a switch port. The switch then relays traffic to and from specific devices across the SAN "Dell N4064F" switch offered from Dell Company can be used to connect our servers to the SAN storage device. The technical datasheet of the switch is available at [20]. "HP 3PAR 7000 2-pt iSCSI/FCoE Adapter (HBA cards))" will be installed in SAN storage device.

After enabling the communications between servers, we need to connect between the internal LANof the faculty and the Datacenter room LAN. in our faculty, we have 120 computer device, each 24 device is connected by cisco Switch, we have number of switches used to connect the computer devices in the faculty building, all these switches are connected by one main manageable switch to connect between the datacenter LAN and the faculty LAN, we need to determine the distance between the main switch in datacenter which connects the servers with each other and the main manageable switch in the faculty building which connects between the switches that connect the computer devices. There is an empty room in the faculty which can be prepared to be a datacenter room. That place is chosen because it is the most secure place in the faculty building. The computer devices and servers will be on the same building so the distance between them is short; they are close to each other. We can connect between the datacenter LAN and the faculty LAN by using fiber optic cables which can connect between the LAN switch in datacenter and the main switch in the faculty LAN. We draw a simple design about our network architecture as follows

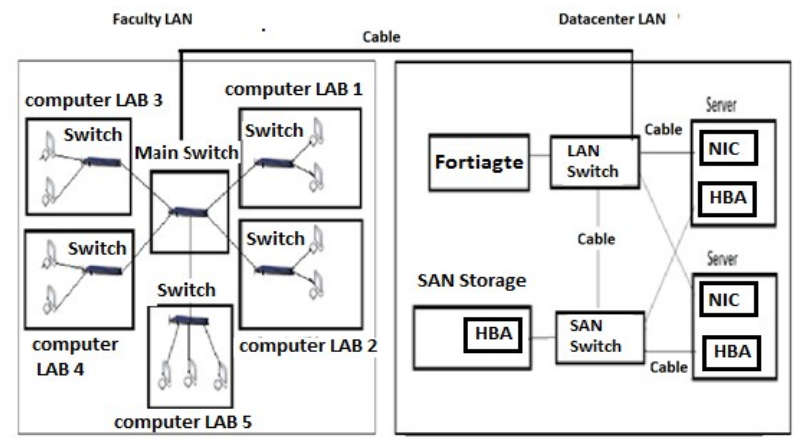

Figure 6. Network Design

For network security, we need to buy security device that monitors incoming and outgoing network traffic and decides whether to allow or block specific traffic based on a defined set of security rules. (FortinetFortiGate 1500DT) product is ideal for protecting our network; it will be connected to the LAN switch, so the switch should have more ports to be able to communicate with other devices. The technical datasheet of the product is available at [21].

The total devices required to build and secure our datacenter LAN are "dell N4064 switch", "dell N4064F switch", patch panels, cables and "FortinetFortiGate 1500DT" product. In our model, we 
International Journal of Computer Science, Engineering and Applications (IJCSEA) Vol. 8, No. 2/3/4, August 2018

only calculate the acquisition cost of the network devices so cables cost, basic installation and maintenance cost of network equipment and other costs related to building and designing the LAN is not included in our model. We now determined the required number of servers and we choose the proper SAN storage and we determined all the required network machines for connecting servers to each other and to the SAN storage device .so we have a complete list of all the required IT devices in our model.

\subsection{UPS AND PDU DEVICES}

To determine the approximate UPS rating our IT devices require, we followed very basic procedure introduced in [22]. First, we made a list of all the equipment our UPS will be protecting then we determine how many volts and amps every device on the list draws and Add all of the VA figures together and finally we multiply the output sum by 1.2 , to build in room for growth. UPS system is connected to PDU units which are used to provide power to all devices housed in racks.

We used the HP Power Advisor tool [23] to estimate the VA rating and the heat load of our servers, SAN storage device and KVM Console Switch. For dell networking switches, we used The Dell Enterprise Infrastructure Planning Tool (EIPT) [24] to know how much heat the switches produce when they are fully loaded and know the VA rating values of switches. About the heat load and VA rating value of "FortiGate-1500DT device", We found in the technical data sheet [18] that at voltage $220 \mathrm{~V}$ the product Heat Dissipation equals to $1,193 \mathrm{BTU} / \mathrm{h}$. and The maximum current is $4 \mathrm{~A}$ at $220 \mathrm{~V}$, so VA $=$ Voltage $*$ current $=4 * 220=880$.

When we used the tools, we first chose the input voltage as 220VAC because the standard voltage in Egypt is 220VAC and then we entered thespecifications of the devices in the tools. We assumed that the devices will be fully utilized to know the maximum heat load that can be produced per hour and the VA rating values. The maximum heat dissipation and VA ratingare the worst-case theoretical maximum numbers provided for planning the infrastructure with fully loaded $100 \%$ traffic, all ports plugged in, and all modules populated. We get the following results:

Table10. IT devices power specifications.

\begin{tabular}{|c|c|c|c|c|}
\hline Product name & $\begin{array}{c}\text { Max Load Input } \\
\text { Power in watts }\end{array}$ & VA Rating & $\begin{array}{c}\text { BTU/ } \\
\text { HR }\end{array}$ & $\begin{array}{c}\text { Syste } \\
\text { m } \\
\text { Curre } \\
\text { nt }\end{array}$ \\
\hline $\begin{array}{c}\text { (HP ProLiant ML350 Gen10 SFF) } \\
\text { One server }\end{array}$ & 707.79 & 712.28 & $2,413.56$ & 3.24 \\
\hline all servers machine (10 servers) & $7,077.9$ & $7,122.8$ & $24,135.6$ & 32.4 \\
\hline $\begin{array}{c}\text { SAN Storage device (HP StoreServ } \\
\text { 7400c 4N) }\end{array}$ & 1031.22 & $1,071.78$ & $3,516.48$ & 4.87 \\
\hline $\begin{array}{c}\text { HPE LCD8500 KVM Console } \\
\text { Switch }\end{array}$ & 59 & 59 & 201.19 & 0.27 \\
\hline dell N4064 switch & 399 & 418 & 1361.4 & 1.9 \\
\hline dell N4064F switch & 206.8 & 220 & 705.6 & 1 \\
\hline FortinetFortiGate 1500DT & 349.8 & 880 & 1,193 & 4 \\
\hline Total value & $9,790.28$ & $9,771.58$ & $31,113.2$ & 44.44 \\
\end{tabular}

Table [10] shows the VA rating of all the IT devices that need to be protected by UPS in our datacenter model. 
International Journal of Computer Science, Engineering and Applications (IJCSEA) Vol. 8, No. 2/3/4, August 2018

The total VA rating of IT devices $=9,771.58=9,771.58 / 1000=9.77158$ KVA. We have to multiply the total by 1.2 to get the grand total. This step accounts for future expansion. The total VA rating of IT devices $=9,771.58 * 1.2=11,725.9 \mathrm{VA}=11,725.9 \mathrm{VA} / 1000=11.7259 \mathrm{KVA}$ rating.

We can use number of PDU devices to distribute power for the different devices. To choose the proper PDU units, we use an online PDU Selector tool offered by HP Company [25]. We enter the value of total VA rating in the tool and the tool shows us the proper solution. For our requirements, the tool shows that" HPE Basic 3.6kVA/60320/C13/WW PDU- H5M56A "product is proper and ideal solution. The technical datasheet is available at [26]

Number of required PDU units for IT devices $=11.7259 \mathrm{KVA}$ rating $/ 3.6 \mathrm{kVA}=3.25=4 \mathrm{PDU}$ devices

We need 4 PDU devices to provide power to all IT devices

UPS Systems must protect all devices which are connected to them in case of power failure and the PDU device itself will be connected to UPS system, we use power advisor tool to calculate the VA rating of the PDU unit, we find the following results

Table 11. PDU power specifications.

\begin{tabular}{|c|c|c|}
\hline Component & One PDU & Four PDU \\
\hline BTU HR & 17.05 BTU & 68.2 \\
\hline VA rating & 5 & 20 \\
\hline
\end{tabular}

UPS Systems must protect all devices which are connected to them in case of power failure and the Number of required PDU units for IT devices $=11.7259 \mathrm{KVA}$ rating $/ 3.6 \mathrm{kVA}=3.25=4 \mathrm{PDU}$ devices

We find that the PDU VA loading is 5VA and we need 4 PDU units for the IT devices so the total VA loading of all PDU units $=5 * 4=20$ VASo we need to add $20 \mathrm{VA}$ to the total VA rating of UPS system. So the total VA rating $=9,771.58+20 \mathrm{VA}=9,791.58$ then we multiply the total by $1.2=9,791.58 * 1.2=11,749.9 \mathrm{VA}=11,749.9 \mathrm{VA} / 1000=11.7499 \mathrm{KVA}$

We searched many times for UPS products on different vendors websites and we find that" HP R/T3000 G4 High Voltage INTL Uninterruptible Power System (J2R04A)" product is ideal for our datacenter. The technical datasheet is available at [27]. We can use number of "HP R3000 (UPS) Uninterruptible Power System". The main feature of the chosen UPS system is that it has high power density with 3300 VA / 3000 Watts. The UPS system can Support up to four (4)" Extended Runtime Modules (ERM)" to increase battery run time during a power outage.

Number of UPS Systems $=$ total VA loading/UPS unit VA load $=11,749.9$ VA/3300VA=3. $5 \sim 4$ UPS systems, so the total required number of the UPS devices necessary for protecting IT devices and PDU units = 4 UPS systems. We used the HP Power Advisor tool [23] to estimate the heat load of our UPS systems and we get the following results in table [11].

Table12. UPS system power specifications

\begin{tabular}{|c|c|c|}
\hline Component & One Ups & 4 UPS \\
\hline BTU HR & 120.71 & 482.84 BTU \\
\hline
\end{tabular}

Number of UPS Systems $=$ total VA loading/UPS unit VA load $=11,749.9$ VA/3300VA=3. $5 \sim 4$ UPS systems, so the total required number of the UPS devices necessary for protecting IT devices and PDU units = 4 UPS systems. We used the HP Power Advisor tool [23] to estimate the heat load of our UPS systems and we get the following results in table [12].

\subsection{Air Conditioning Device}

to calculate the size of air conditioning unit we need for our datacenter room, just add together all the sources of heat and install an air conditioning unit that can remove that much. Providing sufficient cooling is essential to ensure reliable running of IT equipment, UPS and PDU 
International Journal of Computer Science, Engineering and Applications (IJCSEA) Vol. 8, No. 2/3/4, August 2018

equipment. The amount of heat generated is known as the heat gain or heat load. Heat is measured in either British Thermal Units (BTU) or Kilowatts (KW). The heat load depends on a number of factors, by taking into account those that apply in our circumstances and adding them together a reasonably accurate measure of the total heat can be calculated.

The heat gain of a datacenter room depends on the size of the area being cooled, the size and position of windows, and whether they have shading, the number of occupants, the amount of Heat generated by equipment and machinery and the amount of Heat generated by lighting. By calculating the heat gain from each individual item and adding them together, an accurate heat load figure can be determined. In our paper, we only calculate the costs required for cooling the IT devices, UPS systems and PDU units. We already have the value of number of BTU produced form IT devices.

We size our air conditioning for IT devices, UPS systems and PDU units only not for all the datacenter room. We size the air conditioner for sub component in datacenter not for all the complete system in datacenter.

In our model, Table [10] shows the total BTU value of the IT devices including all servers, SAN storage and networking machines. The total BTU value $=31,113.27$ BTU.

In table [11] the total BTU value of PDU devices $=68.2$ BTU. Table [12] shows the total BTU value of our UPS systems $=482.84$ BTU.

We add all the BTU value of IT devices, PDU devices and the UPS system=31,113.27 BTU+68.2 $\mathrm{BTU}+482.84 \mathrm{BTU}=31,664.31 \sim 32000 \mathrm{BTU}$

The total heat load produced from IT devices, UPS systems and PDU units $=31,664.31 \sim 32000$ BTU. We divide the heat load by the cooling capacity of the air conditioning unit in BTU, to determine how many air conditioners are needed.

"Smart Rack 18,000 BTU" Portable Air Conditioning Unit presented by TRIPP.LITE Company can be used in our datacenter to cool the different devices. The technical datasheet of the air conditioning unit is available at [34].

Number of a/c units required $=$ Total heat load BTU / Cooling capacity BTU $=32000$ BTU $/ 18000=1.77 \sim 2$ air conditioning unit.so we need two air conditioner units to cool IT devices and their PDU and UPS systems.

\section{RESULTS AND DiscusSiON 4.1 IT DEVICES COST}

The first component in our cost model is IT devices hardware price. We show in table [12] the different prices of IT devices

\begin{tabular}{|c|c|c|}
\hline Product name & $\begin{array}{l}\text { Final price } \\
\text { from vendors }\end{array}$ & $\begin{array}{c}\text { Warranty } \\
\text { period }\end{array}$ \\
\hline Server machine (HP ProLiant ML350 Gen10 SFF) & $43,680 \$$ & 3 years \\
\hline SAN Storage device (HP StoreServ 7400c 4N) & $31,500 \$$ & 3 years \\
\hline HPE LCD8500 KVM Console Switch & $800 \$$ & 3 years \\
\hline dell N4064 switch & $10,000 \$$ & 3 years \\
\hline dell N4064F switch & $10,000 \$$ & 3 years \\
\hline FortinetFortiGate 1500DT & $18,000 \$$ & 3 years \\
\hline Total devices hardware price & $113,980 \$$ & \\
\hline
\end{tabular}


International Journal of Computer Science, Engineering and Applications (IJCSEA) Vol. 8, No. 2/3/4, August 2018

We called the sales team in HP company to know the current price of the server and told them the server specifications and type of resources and they told us that the price of one server is $5,460 \$$. Total server hardware price $=$ number of servers* server price $=10 * 5,460 \$=54,600 \$$.

The servers costs $54,600 \$$ but they are on sale for $20 \%$ off. $20 \%$ of $54,600 \$=10,920 \$$ so the servers hardware price after the discount rate $=54,600 \$-10,920 \$=43,680 \$$.the KVM Console Switch price is $800 \$$. The SAN Storage device (HP StoreServ 7400c 4N) including all sub components (disk drives, HBA network cards and power supply) price is $31,500 \$$. We have been informed from the DELL Company sales team that the current price of "dell N4064"switch is $10,000 \$$ and the "dell N4064F" switch has the same price. The "FortinetFortiGate 1500DT" Price is $30,000 \$$ so the total IT devices hardware price is $113,980 \$$

\subsection{PDU, UPS AND AIR CONDITIONING COST}

We show in table [13] the cost of the PDU devices required to provide power to IT devices, the cost of the UPS systems required to protect IT devices in case of power failure and the cost of the cooling systems that take the heat away once the power is consumed from IT devices, PDU units and UPS system

Table 14. PDU, UPS and air conditioning hardware price.

\begin{tabular}{|c|c|c|c|}
\hline Component & $\begin{array}{l}\text { Number of } \\
\text { devices }\end{array}$ & $\begin{array}{l}\text { Cost of one } \\
\text { device }\end{array}$ & Total cost \\
\hline PDU units & 4 & $200 \$$ & $800 \$$ \\
\hline $\begin{array}{c}\text { UPS system with Extended } \\
\text { Runtime Modules }\end{array}$ & 4 & $6,301.77 \$$. & $25,207.08 \$$ \\
\hline Air conditioning units & 2 & $4,884.95 \$$ & $9,769.9 \$$ \\
\hline Total cost & & & $35,776.98 \$$ \\
\hline
\end{tabular}

For UPS system ,"HP R/T3000 G4 High Voltage INTL Uninterruptible Power System (J2R04A) "price is \$2,017.37 and "HP R/T3000 G4 Extended Runtime Module" price is 1,071.10 .Each UPS device can support up to four Extended Runtime Modules, so the total price of one UPS system $=\$ 2,017.37+(\$ 1,071 \cdot 10 * 4)=6,301.77 \$$.
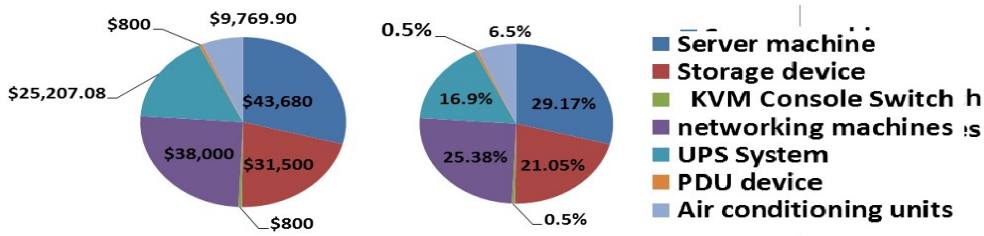

- Air conditioning units

Figure 7. Hardware Costs

Figure [7] shows the hardware costs of IT devices, the PDU devices ,UPS Systems and Airconditioning units in our model .As we see, servers hardware cost are the dominate cost when they are compared with other devices hardware costs. Storage devices and networking machine hardware costs come behind servers hardware cost.

\subsection{Software License Cost (For Server Machines, San Storage Device LICENSE AND FORTIGATE LICENSE) \\ 4.3.1 SOFTWARE LICENSE COST FOR SERVER MACHINES}

Some programs are required to manage the servers and organize the process of transferring data between them as well as managing the process of creating, securing and Operating everything related to the virtual machines. Many companies offer those integrated solutions including what is without a license such as an openstack environment or including a pre-paid license such as the applications presented from Microsoft or IBM companies. Open-source environment is one of the 
International Journal of Computer Science, Engineering and Applications (IJCSEA) Vol. 8, No. 2/3/4, August 2018

most famous environments in the management of virtual machines but it is always under update and somewhat is complicated. Each company has a package of applications that must be purchased to implement the virtual environment on servers and the other IT devices in datacenters. Each company is characterized by the ease of downloading, installing and managing such applications. We show in Table [15] the required applications and their versions in our model

Table 15. Applications and versions.

\begin{tabular}{|c|c|c|}
\hline Software & Version & Total License cost \\
\hline $\begin{array}{c}\text { ESXI } \\
\text { vcenter server }\end{array}$ & 6.5 & $127,894 . \$$ \\
\hline Veeam Backup \& Replication & 9.5 & $14,599.9 \$$ \\
\hline Windows Server & $2012 \mathrm{R} 2$ & $106,462.5 \$$ \\
\hline Oracle DB & $11 \mathrm{~g} \mathrm{R} 2$ & $6,684,500 \$$ \\
\hline Total value & & $6,933,456 \$$ \\
\hline
\end{tabular}

\section{A) WindoWS SERVER 2012 R2 LICENSE}

Windows Server 2012 R2 is Server operating system released by Microsoft. Microsoft allows Customers to choose from three primary editions of Windows Server based on the size of the organization as well as virtualization and datacenter requirements. Editions are Datacenter edition for highly virtualized private cloud environments, Standard edition for non-virtualized or lightly virtualized environments, Essential edition for small businesses with up to 25 users running on servers with up to two processors and Foundation edition for small businesses with up to 15 users running on single processor servers [28]. In our model, we use Datacenter Edition because it is ideal for highly virtualized and software-defined datacenter environments. As always, the Datacenter edition provides access to all the product features and enables unlimited instances of Windows Server with each license, enabling our virtual environment to grow as we do. The licensing for Datacenter edition will continue to be processor plus CAL (Client Access License), with each license covering up to two physical processors on a single server.

There is both a physical and a virtual aspect to these licenses. On the physical side of the equation, a single license of Datacenter will provide us with the right to run the product on up to two physical processors on a single server. On the virtual side of the equation, a license of Datacenter will provide us with the right to run an unlimited number of virtual instances on the licensed physical host.

The MSRP (manufacturer's suggested retail price) of a Datacenter license (without Software Assurance (SA)) though the Open Business program is $\$ 6,156$. In our model, we have 10 servers with 10 processors, A single license will cover two processors, but if and only if those two processors are located in the same physical box. We cannot buy an individual license and assign it to one processor running in one host and another processor running in another host so each processor needs a license

Total License cost $=10 * \$ 6,156=61,560 \$$

Every user or device accessing Windows Server requires a CAL license. We need to purchase a CAL for every device that accesses the server, regardless of the number of users who use that device to access the server. In our model, we have limited number of devices which is 120 computer devices that can access our licensed servers.

The Client Access License based on device cost is $30 \$$ per device In our model, the number of computer devices is 120 so the total CAL cost $=120 * 30=3,600 \$$. Other cost should be added to 
International Journal of Computer Science, Engineering and Applications (IJCSEA) Vol. 8, No. 2/3/4, August 2018

the license cost such as Software Assurance (SA) COST .Software Assurance benefits include new product version rights, deployment planning, technical and end-user training, support, and a unique set of technologies and services.

SA of Server 2012 R2 Datacenter edition license price is 4,130.25\$, we have 10 licensed servers so the total Software Assurance (SA) COST $=4,130.25 * 10=41,302.5 \$$ Total license cost= product license cost $+\mathrm{CAL}$ cost + software assurance $(\mathrm{SA})$ cost $=61,560 \$+3,600 \$+41,302.5$ $\$=106,462.5 \$$

\section{B) ORACLE DB LiCENSE}

Oracle offers customers the option to license by either the Processor metric or the Named User Plus metric [29].

- User based (Named User Plus), Licensed based on a number of users accessing the database whether programmatically or end user

- Processor based, from the name, though we understand it per processor with respect to Oracle, this is different to multi-core processors. Today, every server is running on multicore processors.

Oracle calculates the number of processors on the server as follows:

Number of processors $(\mathrm{P})=$ (number of cores)*(multi-core factor). Each make of processor has their respective multi-core factor.In our model we want to calculate license for Oracle database enterprise edition including support on "Intel ${ }^{\circledR}$ Xeon ${ }^{\circledR}$ Platinum 8180 "processor. we have Number of servers $=10$, each server has one processor with 28 cores.

In oracle, Number of processors $(\mathrm{P})=($ number of cores $) *($ multi-core factor $)=28$ core $* 0.5=14$ processor. We checked the Oracle Processor Core Factor Table [35] and found that power factor of" Intel ${ }^{\circledR}$ Xeon ${ }^{\circledR}$ Platinum 8180 "processor is $(0.5)$. We checked the Oracle Technology Global Price List [30], The MSRP (manufacturer's suggested retail price) of Processor based license is as follows

- $\quad$ EE Oracle database cost $(\mathrm{DB})=47,500 \$$.

- Software update and support price: 10,450\$

Total cost is now calculated as follows:

Number of processors*(enterprise edition Oracle database cost $)+$ Support license cost $=$ $\mathrm{P} *(\mathrm{DB})+\mathrm{SP}=(14 * 47,500 \$)+10,450 \$=658,000 \$+10,450 \$=668,450 \$$ for one server. Total cost for 10 server $=10 * 668,450 \$=6,684,500 \$$

\section{C) VMWARE ESXI}

VMware ESXi is the industry-leading, purpose-built bare-metal hypervisor developed by VMware for deploying and serving virtual servers. ESXi installs directly onto physical server enabling it to be partitioned into multiple logical servers referred to as virtual machines.

We can use ESXi with either the free vSphere Hypervisor or as part of a paid vSphere edition.

A stripped-down free version of ESXi, VMware vSphere Hypervisor, supports fewer features. While it cannot communicate with vCenter Server

While ESXi is itself free, vSphere licensing is required for both the vCenter management server and VMware features. vSphere licensing is available in the different packages (Essentials or Essential Plus Kits) package, (Acceleration Kits) package and (Standard, Enterprise and Enterprise plus Editions for larger environments) package. VMware vSphere with Operations Management Enterprise Plus edition is our choice According to their features. 
International Journal of Computer Science, Engineering and Applications (IJCSEA) Vol. 8, No. 2/3/4, August 2018

vSphere with Operations Management 6.5 is licensed on a per processor basis. Each physical processor (CPU) in a server needs to have at least one processor license key assigned to be able to run vSphere with Operations Management6.5. vSphere with Operations Management 6.5 license keys can be purchased, deployed and managed with No Limits on the Physical Resources of the server, There are no restrictions on physical cores or physical RAM.There are no restrictions on the number of virtual machines that can run on each properly licensed vSphere with Operations Management 6.5 processor [31].

We have more than 4 hosts in our model so we'll need vCenter Server Standard (not vCenter Essentials) edition. The vCenter Standard license is required as an additional purchase. Table [16] shows the license price

Table 16. Vmware license prices.

\begin{tabular}{|c|c|c|c|}
\hline Software & Cost & $\begin{array}{l}\text { \#Physical } \\
\text { Processors }\end{array}$ & $\begin{array}{l}\text { Production } \\
\text { Support }\end{array}$ \\
\hline $\begin{array}{c}\text { VMware vSphere with Operations } \\
\text { Management Enterprise Plus } \\
\text { license }\end{array}$ & $5,294.25 \$$ & 1 & $\$ 1,323.27$ \\
\hline VMware vCenter Server Standard & $\$ 7,224.75$ & & $\$ 1,806.48$ \\
\hline
\end{tabular}

VMware vSphere licenses are purchased on a perpetual basis with basic or Production $24 \times 7$ support. In our model, we have 10 servers with one processor in each one; we add the production support for 24 hour.

For one server, the license cost $=(\$ 5,294.25+\$ 1,323.27)+(\$ 7,224.75+\$ 1,806.48)=12,789.4 \$$

For 10 server, the total license cost $=10 * 12,789.4 \$=127,894 \$$

\section{D) VEEAM ${ }^{\circledR}$ BACKUP \& REPLICATION TM 9.5}

Veeam Backup \& Replication provides unified fast and reliable image-based backup and replication for vSphere virtual environments, giving us the ability to achieve better recovery time and point objectives with VM replication and reduce costs with VM backup, more details about the software can be founded in [32].

Veeam Backup \& Replication licenses are purchased on a perpetual basis with basic or production maintenance or as a subscription license which includes Production $24 \times 7$ support. Veeam Backup \& Replication is licensed per CPU socket on hosts with virtual machines (VMs) to be backed up, replicated, monitored or reported on. A license is required for each motherboard socket as reported by the hypervisor API.

The default method of licensing Veeam products is by the number of sockets on managed servers (hosts). Socket or CPU socket means a physical connector between a microprocessor and a circuit board on a physical server, typically the number of sockets is equal to the number of processors. Veeam ${ }^{\circledR}$ offers different editions of Veeam Availability Suite ${ }^{\mathrm{TM}}$, based on the level of functionality needed from Veeam Backup \& Replication ${ }^{\mathrm{TM}}$. The editions are Standard, Enterprise or Enterprise Plus editions; the comparison between them is discussed at [33].

Veeam Backup \& Replication Enterprise plus License for VMware 1 CPU Socket price + Maintenance \& Support cost $=\$ 1,459.99$. We got the information about the cost from the company sales team. In our model, we have 10 servers with $10 \mathrm{CPU}$ Socket so the total license cost $=1,459.99 \$ * 10=14,599.9 \$$

\subsubsection{SOFTWARE LICENSE COST FOR SAN STORAGE DEVICE}

As mentioned in the technical datasheet of the SAN storage device, two types of software licensing methods are employed with the "HPE 3PAR StoreServ 7000". Some software titles are licensed per system; other titles are licensed by drive. With system-based licensing one license 
International Journal of Computer Science, Engineering and Applications (IJCSEA) Vol. 8, No. 2/3/4, August 2018

covers the whole array, independent of configuration or capacity. Drive-based licensing, in contrast, is licensed per installed drive. A software title with drive-based licensing includes two Licenses to Use (LTU), a Base LTU that enables the software feature for the system and a Drive LTU that licenses the use of one drive. For each software title, the user can purchase one Base LTU per title per array, and one Drive LTU, up to a cap, for every drive, that is installed in the array, independent of drive type. For the "HPE 3PAR StoreServ 7400 "the Drive LTUs cap at 168. After reaching the cap, the users do not need to purchase any more Drive LTUs for that title.In our model, we have one SAN storage device with 40 hard disks.In table [17], we show the SAN Storage License Prices.

Table 17. SAN Storage License Prices.

\begin{tabular}{|c|c|c|c|}
\hline Component & $\begin{array}{l}\text { Software } \\
\text { Suite Base }\end{array}$ & $\begin{array}{l}\text { Software } \\
\text { Suite Drive }\end{array}$ & $\begin{array}{l}\text { Software Suite Drive } \\
\mathbf{* 4 0}\end{array}$ \\
\hline Operating System Software & $1,989.99 \$$ & $1,781.05 \$$ & $71,242 \$$ \\
\hline Security Software Suite & $1,073.99 \$$ & $137.43 \$$ & $6,920 \$$ \\
\hline Data Optimization & $1,660.91 \$$ & $333.10 \$$ & 13,324 \\
\hline $\begin{array}{c}\text { Reporting Software } \\
\text { Application Software Suite for } \\
\text { Oracle }\end{array}$ & $5,476.54 \$$ & & \\
\hline $\begin{array}{c}\text { Application Software Suite for } \\
\text { VMware }\end{array}$ & $6,354.53 \$$ & & \\
\hline $\begin{array}{c}\text { HPE Proactive Care 24x7 } \\
\text { Service }\end{array}$ & & & $2,850 \$$ \\
\hline
\end{tabular}

Total software license cost $=$ (Operating System Software Suite Base + Operating System Software Suite Drive license (1 drive)*40)+ (Security Software Suite Base+ Security Software Suite Drive*40)+ ((Data Optimization Software Suite Base + (Data Optimization Software Suite Drive *40)) + Application Software Suite for Oracle+ Application Software Suite for VMware + HPE Proactive Care 24x7 Service 2,850\$

$=(1,989.99 \$+71,242 \$)+(1,073.99 \$+6,920 \$)+(1,660.91 \$+13,324 \$)+(5,476.54 \$)+(5,476.54 \$)+($ $6,354.53 \$)+2,850 \$=116,368.5 \$$

\subsubsection{SOFTWARE LiCENSE COST FOR NETWORK SWITCHES AND THE ForTigATE FIREWALL}

(Dell Networking OS 6) are operating systems running on the dell switches and they are shipped with the Dell Networking switches without any additional cost.

Licensing on the FortiGate is based on the number of registered clients. FortiGate 1500DT series and higher models support ten (10) free managed FortiClient licenses. For additional managed clients, a FortiClient license subscription must be purchased. In our model, we have more than 10 clients so the FortiClient license subscription must be purchased. As we informed from the sales team that the (FortiClient License Subscription) price is $12,000 \$$ and theFortiGate-1500DT UTM Protection including (24x7 FortiCare plus Application Control, IPS, AV, Botnet IP/Domain and Antispam Services) price is $16,883 \$$.

The total license cost $=12,000 \$+16,883 \$=28,883 \$$.

Servers and other IT devices will be purchased 3 to 5 times during the life of the datacenter. Our plan is to purchase new servers and IT devices at the 3-year mark because that's when the warranty has expired. Extending the warranty can be very expensive, and we may find that replacement parts are not easy to find even when under warranty beyond three years. We found in the technical datasheets of our products that the warranty years are three years for all our IT devices. All our software license contracts are also for three years so they can be compared with each other. After the useful life cycle of the server and other IT devices are finished, we can 
purchase new IT devices but we also have to buy new software license. In figure [8], we show the software license cost in our model
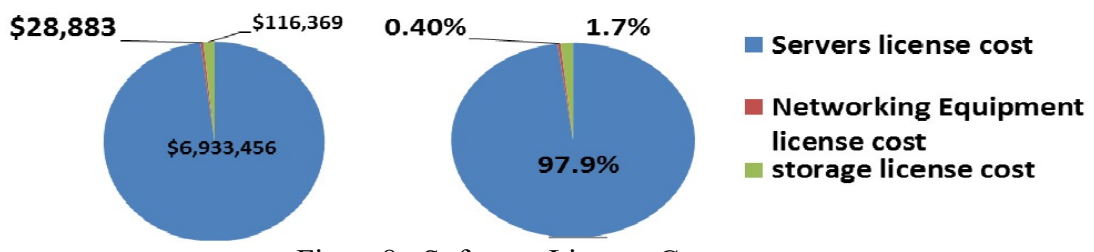

Figure8. Software License Cost

Servers license cost are the dominate cost when compared with storage license cost and networking machines license cost. If we compare between the hardware cost and the software license cost of our IT devices, we found that the software license cost can be greater than the hardware cost of the IT product, for example the hardware price of server machines is $43,680 \$$ but the total software license cost is $6,933,456 \$$.so not only the costs of the hardware component of servers and other IT devices should be taken in consideration by the faculty a administrators when planning to build the datacenter but also the license cost of the software component should be calculated and taken in consideration to obtain the overall datacenter costs.

\section{Conclusion}

This paper presents cost model for data center ownership and operation. Significant costs related to IT devices hardware price, IT software and licensing cost have been discussed together throughout the paper. Our model helps in solving a real problem in faculty of computers and information which has many problems in their computer devices and plans to establish a new datacenter. That cost model can help the faculty administrators to define and know how much money they need to buy the required IT devices and how much IT software license cost. In our cost model, we also calculate the cost of the power distribution equipment required to provide power to IT devices and the cost of the cooling systems that take the heat away once the power is consumed from IT devices, PDU units and UPS systems. The plans for future extensions to our TCO model is to calculate the other costs such as the maintenance cost of IT devices, power consumptions cost, land cost, construction cost and all other costs related to building datacenter for the faculty. We can also make a model to compare between the costs of constructing the datacenter in house or the costs of dealing with cloud providers.

\section{REFERENCES}

[1] C. D. Patel and A. J. Shah, "Cost model for planning, development and operation of a data center," ed, 2005.

[2] J. Karidis, J. E. Moreira, and J. Moreno, "True value: assessing and optimizing the cost of computing at the data center level," in Proceedings of the 6th ACM conference on Computing frontiers, 2009, pp. $185-192$.

[3] J. D. Moore, J. S. Chase, P. Ranganathan, and R. K. Sharma, "Making Scheduling" Cool": Temperature-Aware Workload Placement in Data Centers," in USENIX annual technical conference, General Track, 2005, pp. 61-75.

[4] J. Koomey, K. Brill, P. Turner, J. Stanley, and B. Taylor, "A simple model for determining true total cost of ownership for data centers," Uptime Institute White Paper, Version, vol. 2, p. 2007, 2007.

[5] K. V. Vishwanath, A. Greenberg, and D. A. Reed, "Modular data centers: how to design them?," in Proceedings of the 1st ACM workshop on Large-Scale system and application performance, 2009, pp. 3-10.

[6] J. G. Koomey, "Worldwide electricity used in data centers," Environmental research letters, vol. 3, p. 034008, 2008.

[7] W. P. Turner IV and K. G. Brill, "Cost model: Dollars per $\mathrm{kW}$ plus dollars per square foot of computer floor," Uptime Institute, 2008. 
International Journal of Computer Science, Engineering and Applications (IJCSEA) Vol. 8, No. 2/3/4, August 2018

[8] T. ASHRAE, "Datacom equipment power trends and cooling applications," ed: American Society of Heating, Refrigerating and Air-Conditioning Engineers, Inc. Atlanta, 2005.

[9] N. CRAFT, "Explain: Tier 1 / Tier 2 / Tier 3 / Tier 4 Data Center", nixCraft Website, 29 January 2011," ed.

[10] C. Belady, "Projecting annual new datacenter construction market size," Microsoft, Global Foundation Services Report, 2011.

[11] N. Rasmussen, "Determining total cost of ownership for data center and network room infrastructure," Relatório técnico, Schneider Electric, Paris, vol. 8, 2011.

[12] S. Surabhi, R. Khosla, J. Stern, P. Jashnani, et al., "Oracle Database Installation Guide, 11g Release 2 (11.2) for Microsoft Windows E47798-05."

[13] Veeam Backup \& Replication 9.5 User Guide for VMware vSphere " System Requirements" https://helpcenter.veeam.com/docs/backup/vsphere/system_requirements.html?ver $=95$

[14] C. Zacker, Installing and Configuring Windows Server 2012 R2: Exam 70-410: Wiley, 2014

[15] J. Hamilton, "Overall data center costs," Perspectives http://perspectives.mvdirona.com/2010/09/18/ overalldatacentercosts.aspx. mvdirona. com, 2010.

[16] APC http://www.apc.com/tools/isx/tco/

[17] P. Alto, "Sphere Installation and Setup," Vmware Corporation, 2017.

[18] H. P. Enterprise, "HPE ProLiant ML350 Gen10 Server QuickSpecs (2018)," ed.

[19] H. P. Enterprise, "HPE 3PAR StoreServ 7000 Storage QuickSpecs (2018)," ed.

[20] U. Author, "Dell EMC Networking N4000 series switches," 2017.

[21] U. Author, "FortiGate 1500: FortiGate 1500D, 1500D-DC and 1500DT," 2018.

[22] C. Loeffler and E. Spears, "UPS basics," Eaton Corporation Application Note WP153005EN, pp. 114, 2011.

[23] HPE Power Advisor tool https://paonline56.itcs.hpe.com/?Page=Index

[24] Enterprise infrastructure planning tool http://dell-ui-eipt.azurewebsites.net/\#/

[25] Selector tool for HP PDU http://www.upssizer.com/byLoadPDU_01.asp

[26] H. P. Enterprise, "HPE Basic Power Distribution Units (PDU) QuickSpecs," ed, 2017.

[27] H. P. Enterprise, "HP R/T3000 Uninterruptible Power System (UPS) QuickSpecs," ed, 2011.

[28] U. Author, "Commercial Licensing Reference Guide: Windows Server 2012 R2," 2017.

[29] U. Author, "License Definitions and Rules," .

[30] U. Author, "Oracle Technology Global Price List," 2018.

[31] U. Author, "VMWARE vSPHERE AND vSPHERE WITH OPERATIONS MANAGEMENT: Licensing, Pricing and Packaging," White Paper, 2018.

[32] What's New in Veeam Backup \& Replication 9.5? https://www.veeam.com/whats-new-backupreplication.htm

[33] U. Author, "Veeam Availability Suite," Editions comparison, 2018.

[34] P. Tripp Lite, "SmartRack 18,000 BTU 208/240V Portable Air Conditioning Unit, Server Rooms \& Data Centers, Mfg," Part: SRCOOL18K

[35] U. Author, "Oracle Processor Core Factor Table," 2018.

\section{AUTHORS}

Doaa Bliedy was born in 1991. She received the B.S. degree in Faculty of Computers and Information from Fayoum University, Egypt, in 2012. Her research interests include Datacenter Total costs of ownership. Her E-mail is doaabliedy@gmail.com

Sherif A. Mazen hold a Master degree in Management - from University of Toulouse I France, November 1980. He hold (Ph.D) in Information Systems, from University of Toulouse I, France, December 1982. Now he is Associate Prof. in Information Systems Dept, Faculty of Computers \& Information, Cairo University.Hemay be reached ats.mazen@fcicu.edu.eg

Ehab Ezzat Hassanein hold a Master degree in Computer Science from Northwestern University in 1989.A Ph.D. from Northwestern University in December1992. Now he is Chairman of the Information Systems Department of Computers andInformation Faculty, Cairo University, Egypt. Hemay be reached at e.ezat@fci-cu.edu.eg

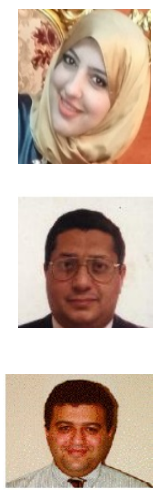

